\title{
Random geometries and real space renormalization group
}

\author{
D.A. Johnston ${ }^{\mathrm{a}}$, J.-P. Kownacki ${ }^{\mathrm{b}}$ and A. Krzywicki ${ }^{\mathrm{b} *}$ \\ ${ }^{a}$ Mathematics Department, Heriot-Watt University, Edinburgh, Scotland \\ bLPTHE, Bâtiment 211, Université Paris-Sud, 91405 Orsay, France
}

\begin{abstract}
A method of "blocking" triangulations that rests on the self-similarity feature of dynamically triangulated random manifolds is proposed and used to define the renormalization group for random geometries. As an illustration, the idea is applied to pure euclidean quantum gravity in 2d. Generalization to more complicated systems and to higher dimensionalities of space-time appears straightforward.
\end{abstract}

We shall report on a work which is still in progress. The aim of this contribution is to share with you the underlying idea.

Although the recent advance in the statistical mechanics of discrete random manifolds has been quite impressive, it appears that our ability to simulate complicated models on a computer exceeds our ability to understand the real significance of the underlying physics (except for a class of $2 \mathrm{~d}$ models). Contrary to discrete field theories where the lattice is only an inert scaffolding, in the case of discrete gravity it is not always evident how to fix the physical scales and how to define the continuum limit. The answers to such questions are usually provided by the renormalization group. One apparently needs to supplement the existing computer artillery with the techniques of the real space renormalization group (RG). In the present context this requires inventing some analogue of Kadanoff's blocking [1] applied to the geometry itself. One suggested approach has been to let coarser versions of an initial triangulation "follow" the original lattice with some appropriate rules as the connectivity is changed during a simulation [2]. In this work we take a different tack, expanding on the ideas put forward in ref. [3]. The dynamical triangulation recipe is adopted.

Conceptually, there are two elements in the familiar Kadanoff construction:

(a) define larger geometrical cells with respect to the smaller ones.

\footnotetext{
${ }^{*}$ Laboratoire associé au C.N.R.S.
}

(b) define "block" spins on larger cells in terms of the spins living on the smaller cells.

On a regular lattice (a) is trivial: the lattice obtained by removing, say, every 2 nd point in each direction is identical modulo rescaling to the original lattice. A change of scale is automatically a self-similarity transformation. On a random lattice (a) is a problem in itself and requires some thought. Therefore, we focus on it here, leaving aside (b) which does not seem to present any serious conceptual difficulty.

We observe that the essential ingredient of the Kadanoff construction is a similarity transformation of the geometry, rather than mere decimation of lattice points (remember that values of critical couplings are lattice dependent). Another important remark is that a randomly triangulated surface is a fractal. This has been emphasized by many people, in particular by Jain and Mathur [4] in their paper on baby universes (BUs), which is particularly relevant to this work. Our idea consists in using this self-similarity to define the step (a). We introduce one additional piece of terminology with respect to [4], namely: BUs with no further BUs growing on them are called last generation BUs. The surface is a hierarchical object so cutting last generation BUs one gets the same ensemble of surfaces modulo rescaling.

Consider $2 \mathrm{~d}$ gravity for simplicity. There is an immediate problem: cutting BUs with neck of length $l$ one creates a polygon which is not necessarily a triangle (it is a triangle when $l=3$, only). This is similar to the problem one encoun- 
ters in Brézin-Zinn-Justin renormalization group program [5], where starting from $\phi^{3}$ one generates all interactions. Thus it would be natural, here also, to consider surfaces made out of arbitrary polygons. Generically, such an ensemble of surfaces is expected to belong to the same universality class as the ensemble of triangulated surfaces, i.e. pure $2 \mathrm{~d}$ gravity. However, although the extension of the model to general cellular decompositions of surfaces does not present any special difficulty, the "BU surgery" becomes rapidly more difficult to implement when the neck length $l$ is larger than 3.

Fortunately, it seems completely sufficient to limit one's attention to BUs with minimum neck length $l=3$, to be called minBUs following the terminology introduced in ref. [4]. This is the choice made in this exploratory paper.

In practice, one must distinguish BUs from a smooth deformation of the surface setting a lower limit on the number of points $B$ in a BU: $B>B_{0}$. It turns out that relevant results are insensitive to the choice of $B_{0}$.

Define $\Delta=\mu_{c}-\mu$, where $\mu$ is the cosmological constant and $\mu_{c}$ is its critical value. Consider an appropriate derivative of the partition function $Z(\Delta)$, to be denoted $\chi(\Delta)$ such that at small $\Delta$

$\chi(\Delta) \sim \int d A A^{\Gamma-1} e^{A \Delta}, \Gamma>0$

The condition $\Gamma>0$ insures that the integral diverges when $\Delta \rightarrow 0$. In generic topology $\chi(\Delta)$ is the so-called string susceptibility and $\Gamma$ is the corresponding critical exponent, conventionally denoted by $\gamma$. In spherical topology, we take an extra derivative of $Z(\Delta)$, so that $\Gamma=\gamma+1$. Neglecting non-singular contribution to the righthand side one has

$\chi(\Delta) \sim \Delta^{-\Gamma}, \Gamma>0$

For pure $2 \mathrm{~d}$ gravity and spherical topology one has exactly $\Gamma=1 / 2$.

We shall now present the results of a computer experiment implementing and illustrating our ideas. We work in spherical topology.

Cutting minBUs produces a mapping of the grand canonical ensemble of surfaces into itself.
We first determine the area distribution of the ensemble of surfaces obtained by cutting last generation minBUs growing out of a (randomly chosen) surface with fixed number of points $A_{0}$, in a sense the "image" of a "point source". We find that this image is a nearly Gaussian curve

$I\left(A \mid A_{0}\right)=\frac{1}{\sqrt{2 \pi \sigma^{2}}} e^{-\left(A-\lambda A_{0}\right)^{2} / 2 \sigma^{2}}[1+\ldots]$

The parameter $\lambda$ becomes rapidly independent of $A_{0}$ and $\sigma^{2}$ increases linearly with $A_{0}$. Higher order cumulants, once scaled by an appropriate power of $\sigma$, are small and seem to decrease with $A_{0}$.

Our experiment has been run with dynamically triangulated surfaces, the number of triangles ranging from 1000 to 20000 . For each value of $A_{0}$ we have carried out a series of several dozens mini-experiments, each mini-experiment corresponding to typically 5000 sweeps of the lattice. About 1000 heating sweeps have been performed between two mini-experiments. The measure of $A$ has been done once every 10 sweeps. The errors have been estimated using the standard binning method.

The exact numerical values of the parameters $\lambda$ and $\sigma$ depend on the choice of the lower cut-off $B_{0}$, defining an "acceptable" minBU. In most of our calculations we have set $B_{0}=10$. An excellent fit to the data is then obtained with $\lambda=$ $0.8189(2)-7.4(4) / A_{0}, \sigma^{2}=2.42(2) A_{0}+185(45)$. Decreasing (increasing) $B_{0}$ one gets, of course, smaller (larger) $\lambda$. For example, with the choice $B_{0}=15$ one finds $\lambda \approx 0.859$. The correction terms in (3) can be organized in a Gram-Charlier series [6]. A very good description of the image is obtained including the first two correction terms in this series. The values of the third and fourth order cumulants for various $A_{0}$ are given in Table 1. The (scaled) cumulants are small and the deviation from the Gaussian is not large, especially at large $A_{0}$.

The image of the full "source" $S\left(A_{0}, \Delta\right) \sim$ $A_{0}^{-1 / 2} \exp \left(A_{0} \Delta\right)$ is given by the convolution

$S^{\prime}(A, \Delta)=\int d A_{0} I\left(A \mid A_{0}\right) S\left(A_{0}, \Delta\right)$ 
Table 1

Scaled third $\left(\kappa_{3}\right)$ and fourth $\left(\kappa_{4}\right)$ order cumulants against $A_{0}$

\begin{tabular}{ccc}
\hline$A_{0}$ & $\kappa_{3} / \sigma^{3}$ & $\kappa_{4} / \sigma^{4}$ \\
\hline 1000 & $-0.50(3)$ & $0.70(14)$ \\
2000 & $-0.33(2)$ & $0.28(3)$ \\
3000 & $-0.24(7)$ & $0.17(11)$ \\
4000 & $-0.22(2)$ & $0.11(5)$ \\
7000 & $-0.11(7)$ & $0.12(11)$ \\
8000 & $-0.21(5)$ & $0.03(13)$ \\
9000 & $-0.21(5)$ & $-0.01(10)$ \\
10000 & $-0.11(4)$ & $0.02(6)$ \\
\hline
\end{tabular}

and can be calculated analytically for large $A$, using the saddle-point method. Keeping only the first term in (3) and remembering that $\sigma^{2} \sim c A_{0}$ one finds for $\Delta \ll 1$

$S^{\prime}(A, \Delta) \sim \frac{1}{\sqrt{\lambda A}} e^{A \Delta / \lambda}$

It is remarkable that the right-hand side does not depend, for small $\Delta$, on the value of the constant $c$. Since the image $S^{\prime}(A, \Delta)$ and the source $S\left(A_{0}, \Delta\right)$ are similar, integrating eq. (5) with respect to $A$ gives the scaling law

$\chi(\Delta)=\lambda^{-\Gamma} \chi\left(\lambda^{-1} \Delta\right), \Gamma=1 / 2$

Since scaled cumulants do not seem to grow with $A_{0}$, the term proportional to the $\mathrm{n}^{\text {th }}$ order Hermite polynomial in the Gram-Charlier series contributes a correction of maximal order $\Delta^{(n-1) / 2}$ to the image $\chi(\Delta)$. Therefore, the corrections do not contribute to the singular part of the image (at least treated term by term).

Although the operation of cutting last generation minBUs is not just a rescaling of the area, we find that the image of a point source is sufficiently sharp for the blocking operation to implement a homogeneous transformation of $\chi(\Delta)$, as long as one is close enough to the critical point. It is clear that the repeated application of this operation defines a renormalization group, in the usual way, so that $\lambda$ in (6) can be replaced by an adjustable scaling factor $\Lambda=\lambda^{n}$.

We expect (6) to hold also for other topologies, when $\Gamma$ differs from $1 / 2$. Obviously, the effective $\Delta \rightarrow 0$ for $\Lambda \rightarrow \infty$. A fully fledged fractal is obtained starting with a smooth surface and repeating the operation of adding minBUs infinitely many times. This corresponds to the limit $\Delta \rightarrow 0$ : being fractal and critical is synonymous.

Generalization to $d>2$ seems conceptually straightforward. In the simplest model one has two independent couplings, the cosmological and the Newton constants, respectively. Thus, instead of the single $\Delta$, there are two such parameters. One can therefore fix independently two dimensionful constants (as it should be for gravity).

In conclusion, we propose how to use the fractal, hierarchical structure of the euclidean spacetime foam to define a real space renormalization group for random geometries. It has been important to check that the idea is meaningful for surfaces of accessible size. In our current work we examine various applications of the idea presented above, focusing on a search for most suitable observables to define the $\beta$ function.

\section{REFERENCES}

1. L.P. Kadanoff, Physics, 2, 263 (1966); for a review see C. Itzykson and J.-M. Drouffe, Statistical Field Theory, Cambridge University Press, Cambridge 1989.

2. R.L. Renken, Univ. of Central Florida preprint (April 1994); R.L. Renken, S. M. Catterall and J. B. Kogut, Univ. of Central Florida preprint (August 1994).

3. A. Krzywicki, unpublished notes (May 1994).

4. S. Jain and S.D. Mathur, Phys. Lett. B286, 239 (1992).

5. E. Brézin and J. Zinn-Justin, Phys. Lett. B288, 54 (1992); S. Higuchi, C. Itoi, S. Nishigaki and N. Sakai, Phys. Lett. B318, 63 (1993).

6. M.G. Kendall and A. Stuart, The Advanced Theory of Statistics, Griffin, London 1963. 
This figure "fig1-1.png" is available in "png" format from: http://arxiv.org/ps/hep-lat/9407018v3 
This figure "fig1-2.png" is available in "png" format from: http://arxiv.org/ps/hep-lat/9407018v3 